\title{
Persistence with antihypertensives in uncomplicated treatment-naïve very elderly patients: a nationwide population-based study
}

Kyung Hee Choi ${ }^{1,5+}$, Yun Mi Yur ${ }^{2+}$, Young-Mi Ah³ ${ }^{3}$ Min Jung Chang ${ }^{4}$ and Ju-Yeun Lee ${ }^{3^{*}}$ (D

\begin{abstract}
Background: Limited studies have evaluated the medication-taking behavior in very elderly hypertensive patients. The aim of this study was to evaluate the persistence and adherence with antihypertensive agents in treatmentnaïve patients, along with other related factors, according to age.

Methods: Adult (19-64 years), elderly (65-79 years), and very elderly ( $\geq 80$ years) uncomplicated hypertensive patients starting antihypertensive monotherapy were identified from the National Health Insurance claims database. The first-year treatment persistence and adherence rates measured using the medication possession ratio were assessed and compared in these three age cohorts.

Results: After propensity score matching, three age cohorts with 6689 patients each were assembled from 228,925 uncomplicated hypertensive patients who began antihypertensive monotherapy in 2012. The treatment persistence and adherence rates over the first year were the lowest in the very elderly (59.5\% and $62.8 \%$, respectively) and highest in the elderly (65.2\% and $67.9 \%$, respectively) patients among the three age cohorts $(p<0.001)$. The adjusted risk for treatment non-persistence was significantly higher in the very elderly (adjusted hazard ratio, 1.20; 95\% confidence interval, 1.13-1.27) compared with the elderly. Having more comorbidities, being a beneficiary of medical aid, and having a diagnosis of dementia were unique positive predictors for treatment persistence in the very elderly, along with common predictors such as female sex, dyslipidemia, and an initially chosen antihypertensive therapeutic class other than beta blockers and thiazide diuretics.

Conclusions: Very elderly patients were less likely to continue antihypertensive therapy over the first year compared with their younger counterparts. Our findings suggest that a low comorbidity index and lack of medical aid support negatively affect the treatment persistence in this population.
\end{abstract}

Keywords: Hypertension, Treatment persistence, Adherence, Aged, Very elderly

\section{Background}

Hypertension is a major health problem worldwide and requires long-term management and continuous therapy. The prevalence among aged people is substantial; approximately $80 \%$ of very elderly patients (defined as those aged over 80 years) have been reported to have

\footnotetext{
* Correspondence: jypharm@hanyang.ac.kr

†Equal contributors

${ }^{3}$ College of Pharmacy, Institute of Pharmaceutical Science and Technology, Hanyang University, 55 Hanyangdaehak-ro, Sangnok-gu, Ansan, Gyeonggi-do 15588, South Korea

Full list of author information is available at the end of the article
}

hypertension [1]. Similarly, according to a report from the National Health Service in England, the prevalence of hypertension was $66.6 \%$ in the population aged over 75 years, as compared with $29.6 \%$ in the overall adult populations in 2014 [2]. As the elderly population increases (especially the very elderly population) [3], the number of patients with chronic diseases requiring longterm care is also increasing. As a result, the healthcare expenditures are increasing worldwide, representing an important social and public health issue [4]. 
Effective management of hypertension in the elderly is vital for preventing disease progression and related comorbidities and for reducing health care costs $[5,6]$. Although hypertension is a principal cause of cardiovascular and cerebrovascular events and is associated with increased mortality [6,7], before the publication of the Hypertension in the Very Elderly Trial, the benefits of treatment in very elderly patients with hypertension were controversial. This trial confirmed that treatment of hypertension reduced mortality even in the very elderly, indicating its importance $[8,9]$.

Age has been considered a potential factor that can influence treatment persistence and adherence [10, 11], and previous studies have mainly focused on elderly populations [12, 13]. In 2008, van Wijk et al. measured persistence with antihypertensive treatment in elderly populations in the US, Canada, and the Netherlands, and found similar patterns of persistence among the three countries [12].

The patterns of medication persistence in Asian population may differ from those in Western countries because of differences in race, healthcare systems, and socioeconomic environment [14, 15]. Different racial and ethnic groups show diverse perceptions and attitudes towards health and medicines, which may affect adherence to therapy [16]. Various reimbursement coverages, drug prices, and financial barriers depending on country-specific healthcare system and socioeconomic environment have been reported to affect medication persistence $[14,15,17]$.

However, very few studies have evaluated hypertensive treatment persistence and adherence in very elderly vs. elderly hypertensive patients in Asia, including Korea. In Korea, the National Health Insurance, a public insurance program operated by the Ministry of Health and Welfare, covers most of the Korean population claims data, including patient data such as the diagnosis, prescription medication, and medical costs, collected by each medical institution. Using these nationwide healthcare claims data in Korea, this study aimed to compare the antihypertensive treatment persistence and adherence patterns in very elderly patients with uncomplicated hypertension to those of matched elderly and adult patients. In addition, we also aimed to identify the factors associated with non-persistence in the very elderly population.

\section{Methods}

\section{Data source and cohort definition}

For this retrospective cohort study, we used the Korean National Health Insurance claims database from January 2011 to December 2013, provided by the Health Insurance Review Agency following data anonymization.

We identified newly diagnosed uncomplicated hypertensive adult patients who started antihypertensive monotherapy in 2012. The index date was defined as the date of the first antihypertensive prescription in 2012. Patients with a diagnosis code of hypertension (International Classification of Diseases [ICD-10] codes I10-13, I15) or who had been prescribed any antihypertensive medication within 1 year before the index date were excluded to ensure the inclusion of only treatment-naïve hypertensive patients. Patients who had been previously diagnosed with the following comorbid conditions were also excluded: cardiovascular disease (I20-I25, I30-I52, Z95), cerebrovascular disease (G45, I60-I69), peripheral vascular disease (I7X), renal disease (N03-N05, N18, N19, Z49, Z94.0, Z99.2), diabetes mellitus (E08-E11, E13), and pregnancy (O00-O9A) [18]. In addition, to evaluate the patients' medication-taking behavior, we excluded patients who had been prescribed only one dose of antihypertensive medication or who had taken the medications for a period of less than 7 days, as well as those who had been hospitalized for more than 7 days within one year from the index date. The patients were followed for one year from the index date and were excluded if their claims data were discontinued before the end of the follow-up period.

The patients were classified into three age categories: adult (19-64 years), elderly (65-79 years), and very elderly ( $\geq 80$ years). The elderly and adult cohorts were constructed using propensity matching with common reference cohorts of the very elderly [18]. The matching variables included sex, the health insurance type, and the Charlson Comorbidity Index [19]. The Charlson Comorbidity Index score, a prognostic comorbidity rating score calculated based on 19 disease states each assigned with a score of $1,2,3$, or 6 , corresponding to the risk of mortality, was assessed using the ICD-10 codes to evaluate the patient's disease burden for 1 year before the index date [19]. The prescribed antihypertensive medications used as the initial therapy were classified by their therapeutic class: angiotensin receptor blockers (ARBs), angiotensin converting enzyme inhibitors (ACEIs), selective beta blockers (BBs), dihydropyridine calcium channel blockers (CCBs), thiazide type diuretics (Ds), alpha blockers (ABs), and others.

The study protocol was approved by the Institutional Review Board (IRB) of Seoul National University (IRB number: P01-201406-SB-03-02). A full ethical review was made for all procedures following the protocol approved by the IRB. The board waived the need for informed consent because only de-identified information was used, without linkable data elements.

\section{Treatment persistence and adherence}

Treatment persistence was measured by the treatment duration, defined as the number of days from the index date to the discontinuation of any antihypertensive treatment or the first appearance of a 60-day prescription gap. 
To assess treatment adherence, we used the medication possession ratio (MPR), calculated as the sum of days covered by any antihypertensive medication one year from the index date divided by 365 days. An MPR $\geq 0.8$ was considered adherent [20].

To examine the patients' age as a factor affecting treatment non-persistence, we calculated the adjusted hazards for treatment non-persistence for the very elderly and elderly cohorts compared with the reference group of adults. We also calculated the adjusted hazard for the very elderly with elderly patients as the reference.

\section{Factors associated with treatment non-persistence}

We assessed the patient sex, Charlson Comorbidity Index score, insurance type, specific underlying diseases (dyslipidemia, dementia, and depression), and the initial therapeutic class of the index therapy as factors potentially affecting treatment non-persistence in the three cohorts. The Charlson Comorbidity Index scores were obtained using the diagnosis codes.

\section{Statistical analysis}

Demographic and clinical characteristics of the three cohorts are presented with descriptive statistics, such as the mean, standard deviation (SD), and percentage. Comparisons of the continuous and discrete data among the three cohorts were performed using analysis of variance (ANOVA) and chi-squared tests, respectively. We considered a $p$ value $\leq 0.05$ to be statistically significant and adjusted this value using Bonferroni correction.

The treatment persistence rates over the first year stratified by the three cohorts were compared using the log-rank test and are presented as adjusted KaplanMeier curves. Multivariate Cox-proportional hazards models were used to evaluate the influence of the potential risk factors on treatment non-persistence. The degree of association is described by the adjusted hazard ratio (aHR) and corresponding 95\% confidence interval (CI). Data management and statistical analyses were performed using SAS 9.3 (SAS Institute, Inc., Cary, NC).

\section{Results}

\section{Patient selection}

We identified 228,925 uncomplicated, treatment-naïve hypertensive patients who began antihypertensive monotherapy in 2012. After excluding patients who were followed-up for less than one year $(n=9364)$, a total of 219,561 patients remained. Of these, 6803 patients (3.1\%) were classified as very elderly and 37,982 patients (17.3\%) as elderly. After propensity score matching for sex, Charlson Comorbidity Index score, and health insurance type, 6689 patients were included in each of the three cohorts.

\section{Demographic and clinical characteristics}

The mean $( \pm$ SD) ages of the adult, elderly, and very elderly cohorts were $50.4( \pm 8.9), 70.9( \pm 4.1)$, and $84.3( \pm$ 4.0) years, respectively. Females comprised $72.6 \%$ of the population in all three cohorts. The mean ( \pm SD) Charlson Comorbidity Index score at baseline was 0.5 ( \pm 0.9 ), and $7.2 \%$ of all patients were receiving medical aid. The number of concomitant medications was the highest in the very elderly cohort and lowest in the adult cohort $(p<0.05)$. Compared with the other two cohorts, the very elderly cohort had the highest proportion of dementia patients and lowest proportion of patients with dyslipidemia $(p<0.0001)$. Depression was more prevalent in the adult cohort than in the other cohorts $(p<0.0001)$. Overall, the most frequent therapeutic class of index therapy was CCBs (48.9\%), followed by ARBs (32.0\%), BBs (10.0\%), and Ds (6.0\%). While this trend was also observed in the very elderly and elderly cohorts individually, ARBs were somewhat more prevalent than CCBs in the adult cohort (Table 1).

\section{Treatment persistence and adherence}

The very elderly cohort showed a significantly shorter treatment duration than the other two cohorts $(p<0.05$, Table 2). Although similar proportions of patients continued antihypertensive treatment after the first three months, the proportion of patients who remained on any antihypertensive treatment at the end of the first year was significantly lower in the very elderly cohort (59.5\%) than in the elderly cohort $(65.2 \%, p<0.001)$. Adjusted Kaplan-Meier analysis of the treatment persistence rate over the first year also revealed lower treatment persistence in the very elderly cohort compared to in the other two cohorts (Fig. 1).

The adherence rate over one year showed a similar pattern, with the lowest rate observed in the very elderly cohort $(62.8 \%$ vs. $64.1 \%$ and $67.9 \%$ in the adult and elderly cohorts, respectively, $p<0.001$ for both) (Table 2).

After adjusting for sex, insurance type, comorbidities, underlying disease, and initial therapeutic class of index therapy, the likelihood of discontinuing antihypertensive treatment was significantly higher in the very elderly patients (aHR, 1.20; 95\% CI, 1.13-1.27) compared with in the elderly patients. However, the adjusted risk of treatment non-persistence in the very elderly (aHR, 0.99; 95\% CI, 0.94-1.05) was similar to that of adults (aHR, 0.99; 95\% confidence interval (CI), 0.94-1.05), while elderly patients exhibited a significantly lower risk than adults (aHR, 0.84; 95\% CI, 0.79-0.89).

\section{Predictors of treatment non-persistence}

Table 3 shows the predictors of treatment nonpersistence in the three cohorts. Although the presence of comorbidities was not significantly associated 
Table 1 Demographic and clinical characteristics of the propensity-matched cohort

\begin{tabular}{|c|c|c|c|c|c|c|}
\hline Characteristics & $\begin{array}{l}\text { Total } \\
(n=20,067)\end{array}$ & $\begin{array}{l}\text { Adults }^{\mathrm{a}} \\
(n=6689)\end{array}$ & $\begin{array}{l}\text { Elderly }{ }^{\mathrm{b}} \\
(n=6689)\end{array}$ & $\begin{array}{l}\text { Very elderly }{ }^{c} \\
(n=6689)\end{array}$ & $p$ value ${ }^{*}$ & $\begin{array}{l}\text { Post } \\
\text { hoc }^{+}\end{array}$ \\
\hline Age, years, mean (SD) & $68.5(15.2)$ & $50.4(9.0)$ & $70.9(4.1)$ & $84.3(4.0)$ & $<0.0001$ & $a<b<c$ \\
\hline Sex, male, n (\%) & $5502(27.4)$ & $1834(27.4)$ & $1834(27.4)$ & $1834(27.4)$ & 1.000 & \\
\hline $\begin{array}{l}\text { Charlson comorbidity } \\
\text { index score, mean (SD) }\end{array}$ & $0.5(0.9)$ & $0.5(0.9)$ & $0.5(0.8)$ & $0.5(0.9)$ & 0.948 & \\
\hline 0 & $12,780(63.7)$ & $4258(63.7)$ & $4264(63.8)$ & $4258(63.7)$ & 1.000 & \\
\hline$\geq 1$ & $7287(36.3)$ & $2431(36.3)$ & $2425(36.3)$ & $2431(36.3)$ & 0.992 & \\
\hline \multicolumn{7}{|l|}{ Health insurance type } \\
\hline Health insurance & $18,621(92.8)$ & 6207 (92.8) & $6207(92.8)$ & 6207 (92.8) & 1 & \\
\hline Medical aid & $1446(7.2)$ & $482(7.2)$ & $482(7.2)$ & $482(7.2)$ & & \\
\hline $\begin{array}{l}\text { Number of concomitant } \\
\text { medications, mean (SD) }\end{array}$ & $3.3(2.8)$ & $2.7(2.4)$ & $3.4(2.8)$ & $3.7(3.0)$ & $<0.0001$ & $\mathrm{a}<\mathrm{b}<\mathrm{c}$ \\
\hline \multicolumn{7}{|l|}{ Underlying disease, n (\%) } \\
\hline Dementia & $277(2.1)$ & $24(0.2)$ & $59(0.9)$ & $194(2.9)$ & $<0.0001$ & $\mathrm{a}<\mathrm{b}<\mathrm{c}$ \\
\hline Depression & $639(4.6)$ & 440 (3.2) & 104 (1.6) & $95(1.4)$ & $<0.0001$ & $a>b, c$ \\
\hline Dyslipidemia & $2496(16.3)$ & $1963(12.8)$ & $366(5.5)$ & $167(2.5)$ & $<0.0001$ & $a>b>c$ \\
\hline \multicolumn{7}{|l|}{ Therapeutic class of index therapy, n (\%) } \\
\hline $\begin{array}{l}\text { Dihydropyridine calcium channel } \\
\text { blockers }\end{array}$ & 9807 (48.9) & 2619 (39.2) & $3374(50.4)$ & $3814(57.0)$ & $<0.0001$ & $<0.0001$ \\
\hline Angiotensin receptor blockers & $6419(32.0)$ & $2722(40.7)$ & $2077(31.1)$ & $1620(24.2)$ & & \\
\hline Selective beta blockers & 2009 (10.0) & $900(13.5)$ & $620(9.3)$ & $489(7.3)$ & & \\
\hline Thiazide type diuretics & $1212(6.0)$ & $324(4.8)$ & $412(6.2)$ & $476(7.1)$ & & \\
\hline Alpha blockers & 359 (1.8) & $29(0.4)$ & $128(1.9)$ & $202(3.0)$ & & \\
\hline $\begin{array}{l}\text { Angiotensin converting enzyme } \\
\text { inhibitors }\end{array}$ & $250(1.2)$ & $84(1.3)$ & $78(1.2)$ & $88(1.3)$ & & \\
\hline Others & $11(0.1)$ & $11(0.2)$ & $0(0.0)$ & $0(0.0)$ & & \\
\hline
\end{tabular}

"Analysis of variance test or chi-squared test of the three groups

${ }^{\dagger}$ Bonferroni correction $(p<0.015)$ with student's t-test or chi-squared test

${ }^{\mathrm{a}}$ Adult cohort ${ }^{\mathrm{b}}$ Elderly cohort ${ }^{\mathrm{C}}$ Very elderly cohort

with treatment persistence in uncomplicated adult or elderly hypertensive patients, patients with at least one comorbidity were more likely to continue treatment in the very elderly cohort (aHR, 0.93; 95\% CI, 0.87-0.98). Among the very elderly, patients who received medical aid were more likely to persist with the treatment (aHR, 0.83; 95\% CI, 0.71-0.98), while in the adult cohort, those receiving medical aid were at higher risk of non-persistence (aHR, 1.21; 95\% CI, 1.06-1.39). Patients with dementia showed a lower

Table 2 Comparison of treatment persistence and adherence among propensity-matched cohorts

\begin{tabular}{|c|c|c|c|c|c|}
\hline Variables & Adults ${ }^{a}(n=6689)$ & Elderly $^{\mathrm{b}}(n=6689)$ & Very elderly ${ }^{c}(n=6689)$ & $p$-value & Post hoc ${ }^{\dagger}$ \\
\hline Treatment duration, days, mean (SD) & $277.1(122.8)$ & $280.6(123.3)$ & $267.6(127.2)$ & $<0.0001$ & $a, b>c$ \\
\hline \multicolumn{6}{|l|}{ Treatment persistence rate, $\mathrm{n}(\%)$} \\
\hline at the first 3 months & $5753(86.0)$ & $5764(86.2)$ & $5663(84.7)$ & 0.024 & $b>c$ \\
\hline at the first 6 months & $4731(70.7)$ & $4754(71.1)$ & $4473(66.9)$ & $<0.0001$ & $a, b>c$ \\
\hline at the first 9 months & $4422(66.1)$ & $4532(67.8)$ & $4207(62.9)$ & $<0.0001$ & $a, b>c$ \\
\hline at the first 12 months & $4179(62.5)$ & $4364(65.2)$ & 3979 (59.5) & $<0.0001$ & $b>a>c$ \\
\hline Medication possession ratio, mean (SD) & $0.76(0.30)$ & $0.79(0.29)$ & $0.75(0.30)$ & $<0.0001$ & $b>a, c$ \\
\hline Treatment adherence rate, $\mathrm{n}(\%)$ & $4290(64.1)$ & $4542(67.9)$ & $4135(62.8)$ & $<0.0001$ & $b>a>c$ \\
\hline
\end{tabular}




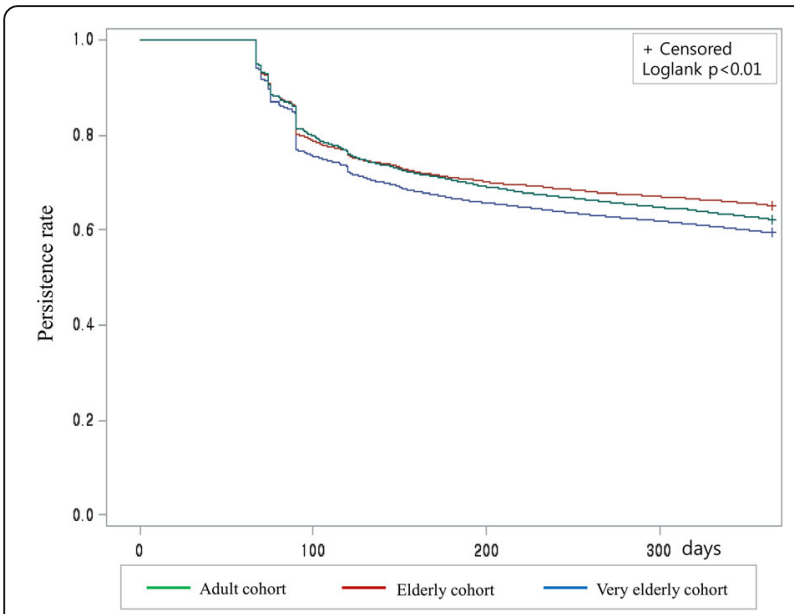

Fig. 1 Treatment persistence rate over the first year among adult, elderly and very elderly cohorts $(n=20,067)$. Green line: adults (1964 years); Red line: elderly (65-79 years); Blue line: very elderly ( $\geq 80$ years) risk of discontinuing treatment only in the very elderly cohort (aHR, 0.68; 95\% CI, 0.52-0.90). Finally, in terms of the initial therapeutic classes of drugs, only Ds and BBs were associated with treatment non-persistence in elderly and very elderly patients (Table 3).

\section{Discussion}

To our knowledge, this is the first nationwide populationbased study focused on antihypertensive medication persistence and adherence in a very elderly population at the time of initiation of antihypertensive monotherapy in Korea. We found that very elderly patients were more likely to discontinue antihypertensive treatment than elderly or adult patients, and that the predictors of treatment non-persistence in the very elderly differed from those in the other age groups. The one-year treatment persistence rate in very elderly patients was significantly lower than that in elderly patients $(59.5 \%$ vs. $65.2 \%, p<0.001)$; after adjustment for potential confounding variables, very elderly patients remained at elevated risk of treatment nonpersistence compared with elderly patients (aHR, 1.20; 95\% CI, 1.13-1.27). Despite the statistically significant differences in treatment persistence and adherence among

Table 3 Predictors for antihypertensive treatment non-persistence in three propensity-matched cohorts ${ }^{a}$

\begin{tabular}{|c|c|c|c|c|c|c|}
\hline \multirow[t]{2}{*}{ Characteristics } & \multicolumn{2}{|c|}{ Adults $(n=6689)$} & \multicolumn{2}{|c|}{ Elderly $(n=6689)$} & \multicolumn{2}{|c|}{ Very elderly $(n=6689)$} \\
\hline & $\mathrm{aHR}$ & $95 \% \mathrm{Cl}$ & $\mathrm{aHR}$ & $95 \% \mathrm{Cl}$ & $\mathrm{aHR}$ & $95 \% \mathrm{Cl}$ \\
\hline \multicolumn{7}{|l|}{ Sex } \\
\hline Male (reference) & 1.00 & & 1.00 & & 1.00 & \\
\hline Female & 0.90 & $0.82-0.98$ & 0.92 & $0.84-1.01$ & 0.85 & $0.78-0.93$ \\
\hline \multicolumn{7}{|l|}{ Charlson comorbidity index score } \\
\hline 0 (reference) & 1.00 & & 1.00 & & 1.00 & \\
\hline$\geq 1$ & 0.95 & $0.87-1.03$ & 0.98 & $0.92-1.04$ & 0.93 & $0.87-0.98$ \\
\hline \multicolumn{7}{|l|}{ Health insurance type } \\
\hline Health insurance (reference) & 1.00 & & 1.00 & & 1.00 & \\
\hline Medical aid & 1.21 & $1.06-1.39$ & 1.07 & $0.92-1.26$ & 0.83 & $0.71-0.98$ \\
\hline \multicolumn{7}{|l|}{ Underlying disease risk factor } \\
\hline Dementia & 1.66 & $0.93-2.97$ & 1.15 & $0.75-1.77$ & 0.68 & $0.52-0.90$ \\
\hline Depression & 1.11 & $0.95-1.28$ & 1.36 & $1.00-1.85$ & 0.91 & $0.63-1.32$ \\
\hline Dyslipidaemia & 0.64 & $0.59-0.71$ & 0.75 & $0.61-0.92$ & 0.75 & $0.57-0.98$ \\
\hline \multicolumn{7}{|l|}{ Therapeutic class of index therapy } \\
\hline Angiotensin receptor blockers (reference) & 1.00 & & 1.00 & & 1.00 & \\
\hline Dihydropyridine calcium channel blockers & 1.12 & $1.02-1.22$ & 1.03 & $0.93-1.13$ & 1.04 & $0.94-1.14$ \\
\hline Selective beta blockers & 1.93 & $1.73-2.16$ & 1.58 & $1.37-1.82$ & 1.29 & $1.11-1.51$ \\
\hline Thiazide diuretics & 4.04 & $3.49-4.67$ & 2.87 & $2.48-3.33$ & 1.74 & $1.51-2.02$ \\
\hline Alpha blockers & 1.90 & $1.15-3.15$ & 1.23 & $0.92-1.65$ & 1.03 & $0.81-1.30$ \\
\hline Angiotensin converting enzyme inhibitors & 1.42 & $1.03-1.97$ & 1.14 & $0.77-1.68$ & 0.96 & $0.68-1.37$ \\
\hline Others & 2.39 & $1.18-4.88$ & & & & \\
\hline
\end{tabular}

Abbreviations: aHR adjusted hazard ratio; $\mathrm{Cl}$ confidence interval

a Predictors for antihypertensive treatment non-persistence were identified using a multivariate Cox-proportional Hazard model 
the three age cohorts, the one-year treatment adherence was more than $60 \%$ in all three groups. These results corroborate the findings from other studies conducted in Western countries $[12,13]$. In 2008, van Wijk et al. reported that approximately $25 \%$ of elderly patients studied were non-persistent, defined as a treatment gap of at least 180 days during the first year of treatment, with little difference among the three examined countries (the US, Canada, and the Netherlands) [12]. In 2014, Tu et al. found that $64.6 \%$ of elderly patients in Canada were therapy-persistent for over one year [13].

The present study found that elderly patients aged 6579 years were more persistent with antihypertensive treatment than adults, while the very elderly showed poor treatment persistence. This result is consistent with previously published results from Canada [13] and Sweden [21], which showed reduced odds of medication persistence in patients older than 75 years compared to those aged 66-70 years. However, other previous studies that have investigated the impact of aging on treatment persistence have reached conflicting results [22, 23]. These discrepancies may be partially explained by differences in age composition, reference groups, and methodology used in measuring persistence, such as a prescription gap, between the studies.

Unlike in previous studies, the present study included only uncomplicated hypertensive patients. Therefore, the Charlson Comorbidity Index score was low, and twothirds of the study patients had no comorbid conditions. The findings from previous studies investigating comorbidities as a risk factor for treatment non-persistence have been inconsistent $[13,23]$. In the present investigation, we found that patients with one or more comorbidities were significantly more likely to remain treatment persistent than those without a comorbidity in the very elderly population, whereas the presence of comorbidities did not predict poor persistence in adults or in the elderly. These findings may be explained by the fact that very elderly patients with comorbidities might be more likely to continue hypertensive treatment because they require regular visits to healthcare facilities for diseases other than hypertension. Furthermore, in the very elderly population, patients with comorbidities might be more likely to continue hypertensive treatment because they require regular visits to healthcare facilities for diseases other than hypertension.

Beneficiaries of medical aid receive prescription drugs free of charge in Korea. Accordingly, in our study, being a beneficiary of medical aid was positively associated with treatment persistence in the very elderly cohort (aHR, 0.83; 95\% CI, 0.71-0.98). This finding is consistent with those of several studies that reported sufficient health insurance or prescription drug coverage as the principal contributing factor to treatment persistence
$[10,14,24]$. On the other hand, in our study, the insurance type was negatively associated with treatment persistence in the adult cohort (aHR: 1.21; 95\% CI: $1.06-$ 1.39). This finding may be explained by the difference in eligibility for medical aid between the adult and very elderly populations in Korea. Adults who receive medical aid in Korea tend to have a very low economic status, which is a recognized risk factor for lower treatment persistence [14, 25].

Moreover, very elderly patients diagnosed with dementia or dyslipidemia were significantly more likely to remain persistent with antihypertensive treatment. The finding of the association between dyslipidemia and treatment persistence corroborates a number of published studies [12, 23, 25, 26]. However, our finding of higher persistence in very elderly patients with dementia contrasts with the results from several previous studies reporting a higher discontinuation rate for antihypertensive treatment among patients with dementia or cognitive impairment $[27,28]$. These contradictory findings are somewhat puzzling, and may be partially explained by the lower medication complexity in older adults with cognitive impairment, as observed in one previous study [29]. The care management by caregivers may also increase the treatment persistence in the very elderly with dementia [30], which is supported by the findings of recent studies that dementia was associated with the likelihoods of continuing statin therapy (aHR, 0.84; 95\% CI, $0.73-0.98)$ and antiplatelet therapy in elderly patients after ischemic stroke [31, 32].

Ds and BBs as initial antihypertensives were negatively associated with treatment persistence, as compared with ARBs, in the very elderly cohort, whereas the rate of non-persistence in the adult cohort was slightly but significantly higher for $\mathrm{CCB}, \mathrm{AB}$, and ACEI initiators, as well as $B B$ and $D$ initiators, as compared with ARB initiators. The Eighth Joint National Committee guidelines do not recommend BBs for initial hypertensive therapy due to the relatively high risks of cardiovascular death, myocardial infarction, and stroke with these agents. In addition, the Canadian Institute for Health Information reported that Ds associate with the lowest persistence when newly prescribed in patients over 65 years of age $[33,34]$. Marcum et al. analyzed the rate of hospitalization from adverse drug reactions in older veterans and found that BBs and Ds were associated with significantly elevated rates [35]. Thus, the low persistence commonly observed in elderly and very elderly patients when beginning a $\mathrm{BB}$ or $\mathrm{D}$ may stem from the higher risk of adverse drug reactions from these antihypertensive classes.

The increased risk of adverse drug events and inconsistency in the efficacy associated with changes in pharmacokinetic and pharmacodynamic profiles with aging 
also may negatively affect drug persistence particularly in the very elderly compared with the elderly patients [36, 37].

Some limitations should be considered when interpreting our findings. First, due to the nature of retrospective claims data, we could not confirm whether the patients actually took the medications that were prescribed. Therefore, our study likely overestimated the actual treatment adherence and persistence. Second, the study only included patients who started monotherapy for uncomplicated hypertension. We excluded patients who began combination therapy in order to minimize confounding by disease severity, which could not be reliably measured in our cohort. Therefore, our results may not be generalizable to patients administered combination therapy. Finally, while we measured the health insurance type as a marker of socioeconomic position, we could not consider other important socioeconomic variables due to limitations of the claims data.

\section{Conclusions}

This study showed that very elderly patients were more likely to discontinue antihypertensive treatment when compared with elderly patients; approximately $40 \%$ of very elderly uncomplicated hypertensive patients were treatment non-persistent during the first year. In addition, this study suggests that a low comorbidity index and lack of medical aid support negatively affect the treatment persistence uniquely in this population.

\section{Abbreviations \\ Abs: Alpha blockers; ACEls: Angiotensin converting enzyme inhibitors; aHR: Adjusted hazard ratio; ANOVA: Analysis of variance; ARB: Angiotensin receptor blockers; BBs: Selective beta blockers; CCBs: Dihydropyridine calcium channel blockers; Cl: Confidence interval; Ds: Thiazide type diuretics; ICD: International classification of diseases; IRB: Institutional review board; MPR: Medication possession ratio; SD: Standard deviation}

\section{Acknowledgements}

Not Applicable.

Funding

None.

\section{Availability of data and materials}

The datasets used and/or analysed during the current study are available from the corresponding author on reasonable request.

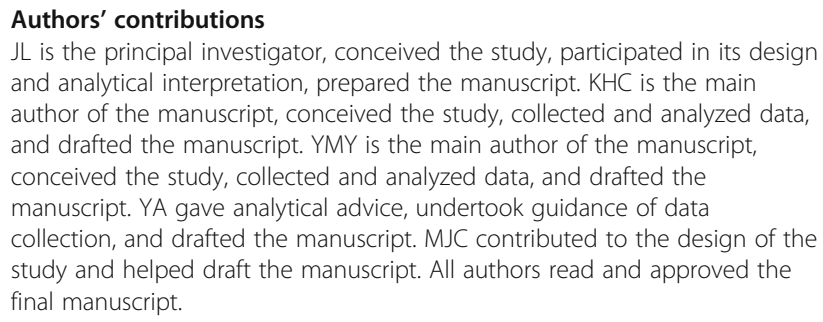

\section{Ethics approval and consent to participate}

This study protocol was approved by the Institutional Review Board (IRB) of Seoul National University (IRB number: P01-201406-SB-03-02). A full ethical review was made for all procedures following the protocol approved by the IRB. The board waived informed consent because only de-identified information was used without linkable data elements.

Consent for publication

Not applicable.

\section{Competing interests}

The authors declare that they have no competing interests.

\section{Publisher's Note}

Springer Nature remains neutral with regard to jurisdictional claims in published maps and institutional affiliations.

\section{Author details \\ ${ }^{1}$ College of Pharmacy, Sunchon National University, 255 Jungang-ro, Suncheon, Jeollanam-do 57922, South Korea. ${ }^{2}$ College of Pharmacy \& Research Institute of Pharmaceutical Sciences, Seoul National University, 103 Daehak-ro, Jongno-gu, Seoul 03080, South Korea. ${ }^{3}$ College of Pharmacy, Institute of Pharmaceutical Science and Technology, Hanyang University, 55 Hanyangdaehak-ro, Sangnok-gu, Ansan, Gyeonggi-do 15588, South Korea. ${ }^{4}$ College of Pharmacy and Yonsei Institute of Pharmaceutical Sciences, Yonsei University, Incheon 21983, South Korea. ${ }^{5}$ College of Pharmacy, Chonnam National University, Gwang-Ju 61186, South Korea.}

Received: 13 March 2017 Accepted: 16 August 2017

Published online: 24 August 2017

\section{References}

1. Cohen DL, Townsend RR. Update on pathophysiology and treatment of hypertension in the elderly. Curr Hypertens Rep. 2011;13:330-7.

2. Health Survey for England. England National Health Service. 2014; http:// content.digital.nhs.uk/article/2021/Website-Search?productid $=19587 \& q=$ Health+Survey+for+England\&sort=Relevance\&size=10\&page=1\&area=both. Accessed 07 Nov 2016

3. World health report: health of the elderly. World Health Organization. http:/ www.who.int/whr/1995/media_centre/executive_summary1/en/index4.html. Accessed 07 Nov 2016.

4. Getzen TE. Population aging and the growth of health expenditures. J Gerontol. 1992:47:S98-104.

5. Krittayaphong R, Rangsin R, Thinkhamrop B, Hurst C, Rattanamongkolgul S, et al. Prevalence and associating factors of atrial fibrillation in patients with hypertension: a nation-wide study. BMC Cardiovasc Disord. 2016;16:57.

6. Richman IB, Fairley M, Jorgensen ME, Schuler A, et al. Cost-effectiveness of intensive blood pressure management. JAMA cardiology. 2016;1:872-9.

7. Tadic M, Cuspidi C, Hering D. Hypertension and cognitive dysfunction in elderly: blood pressure management for this global burden. BMC Cardiovasc Disord. 2016;16:208

8. Beckett $\mathrm{N}$, Peters $\mathrm{R}$, Tuomilehto J, et al. Immediate and late benefits of treating very elderly people with hypertension: results from active treatment extension to hypertension in the very elderly randomised controlled trial. BMJ. 2012;344:d7541.

9. Beckett NS, Peters R, Fletcher AE, et al. Treatment of hypertension in patients 80 years of age or older. N Engl J Med. 2008;358:1887-98.

10. Klootwyk J, Sanoski C. Medication adherence and persistence in hypertension management. J Clin Outcomes Manage. 2011;18:351-8.

11. Balkrishnan R. Predictors of medication adherence in the elderly. Clin Ther. 1998:20:764-71.

12. van Wijk BL, Shrank WH, Klungel OH, et al. A cross-national study of the persistence of antihypertensive medication use in the elderly. J Hypertens. 2008;26:145-53.

13. Tu K, Anderson LN, Butt DA, et al. Antihypertensive drug prescribing and persistence among new elderly users: implications for persistence improvement interventions. Can J Cardiol. 2014;30:647-52.

14. Elliott WJ. Improving outcomes in hypertensive patients: focus on adherence and persistence with antihypertensive therapy. J Clin Hypertens. 2009;11:376-82.

15. Lee SY, Chun CB, Lee YG, Seo NK. The National Health Insurance system as one type of new typology: the case of South Korea and Taiwan. Health policy. 2008:85:105-13. 
16. Chong E, Wang H, King-Shier KM, et al. Prescribing patterns and adherence to medication among south-Asian, Chinese and white people with type 2 diabetes mellitus: a population-based cohort study. Diabet Med. 2014;12: 1586-93.

17. Maxwell CJ, Stock K, Seitz D, et al. Persistence and adherence with dementia pharmacotherapy: relevance of patient, provider, and system factors. Can J Psychiatr. 2014;59:624-31.

18. Tinetti ME, Han L, Lee DS, McAvay GJ, Peduzzi P, Gross CP, Zhou B, Lin H. Antihypertensive medications and serious fall injuries in a nationally representative sample of older adults. JAMA Intern Med. 2014;174:588-95.

19. Charlson ME, Pompei P, Ales KL, MacKenzie CR. A new method of classifying prognostic comorbidity in longitudinal studies: development and validation. J Chronic Dis. 1987;40:373-83.

20. Ah YM, Lee JY, Choi YJ, et al. Persistence with antihypertensive medications in uncomplicated treatment-Nairve patients: effects of initial therapeutic classes. J Korean Med Sci. 2015;30:1800-6.

21. Hedna K, Hakkarainen KM, Gyllensten H, et al. Adherence to antihypertensive therapy and elevated blood pressure: should we consider the use of multiple medications? PLoS One. 2015;10:e0137451.

22. Baggarly SA, Kemp RJ, Wang X, Magoun AD. Factors associated with medication adherence and persistence of treatment for hypertension in a Medicaid population. Res Social Adm Pharm. 2014;10:e99-112.

23. Friedman $\mathrm{O}, \mathrm{McAlister} F A$, Yun $L$, et al. Antihypertensive drug persistence and compliance among newly treated elderly hypertensives in ontario. Am J Med. 2010;123:173-81.

24. Tajeu GS, Kent ST, Kronish IM, Huang L, Krousel-Wood M, Bress AP, Shimbo $D$, Muntner P. Trends in antihypertensive medication discontinuation and low adherence among Medicare beneficiaries initiating treatment from 2007 to 2012. Hypertension. 2016;68:565-75.

25. Borghi C, Veronesi M, Dormi A, et al. Persistence of treatment and blood pressure control in elderly hypertensive patients treated with different classes of antihypertensive drugs. Am J Geriatr Cardiol. 2007;16:280-6.

26. Agarwal S, Tang SS, Rosenberg N, et al. Does synchronizing initiation of therapy affect adherence to concomitant use of antihypertensive and lipidlowering therapy? Am J Ther. 2009;16:119-26.

27. Cooney D, Pascuzzi K. Polypharmacy in the elderly: focus on drug interactions and adherence in hypertension. Clin Geriatr Med. 2009:25:221-33.

28. Mancia G, Zambon A, Soranna D, et al. Factors involved in the discontinuation of antihypertensive drug therapy: an analysis from real life data. J Hypertens. 2014;32:1708-15.

29. Lee DS, de Rekeneire N, Hanlon JT, Gill TM, Bauer DC, Meibohm B, Harris TB, Jeffery SM. Cognitive impairment and medication complexity in community-living older adults: the health, aging and body composition study. J Pharm Technol. 2012;28:156-62

30. Arlt S, Lindner R, Rosler A, von Renteln-Kruse W. Adherence to medication in patients with dementia: predictors and strategies for improvement. Drugs Aging. 2008;25:1033-47.

31. Wawruch M, Zatko D, Wimmer G Jr, Luha J, Hricak V, et al. Patient-related characteristics associated with non-persistence with statin therapy in elderly patients following an ischemic stroke. Pharmacoepidemiol Drug Saf. 2017; 26:201-7.

32. Wawruch M, Zatko D, Wimmer G Jr, Luha J, Kuzelova L, et al. Factors influencing non-persistence with antiplatelet medications in elderly patients after Ischaemic stroke. Drugs Aging. 2016;33:365-73.

33. James PA, Oparil S, Carter BL, et al. 2014 evidence-based guideline for the management of high blood pressure in adults: report from the panel members appointed to the eighth joint National Committee (JNC 8). JAMA. 2014;311:507-20.

34. Persistence and compliance with cardiovascular drug therapy among seniors. Canadian Institude for Health Information. https://secure.cihi.ca/ free_products/PersistenceCompliance_CardiovascularDrugTherapy_Seniors_ EN.pdf. Accessed 07 Nov 2016.

35. Marcum ZA, Amuan ME, Hanlon JT, et al. Prevalence of unplanned hospitalizations caused by adverse drug reactions in older veterans. J Am Geriatr Soc. 2012;60:34-41.

36. Sera LC, McPherson ML. Pharmacokinetics and pharmacodynamic changes associated with aging and implications for drug therapy. Clin Geriatr Med. 2012;28:273-86.

37. Hilmer SN. ADME-tox issues for the elderly. Expert Opin Drug Metab Toxicol. 2008:4:1321-31.

\section{Submit your next manuscript to BioMed Central and we will help you at every step:}

- We accept pre-submission inquiries

- Our selector tool helps you to find the most relevant journal

- We provide round the clock customer support

- Convenient online submission

- Thorough peer review

- Inclusion in PubMed and all major indexing services

- Maximum visibility for your research

Submit your manuscript at www.biomedcentral.com/submit
Biomed Central 